\title{
Validación del cuestionario de medida del conocimiento del paciente sobre su medicamento adaptado al portugués
}

\author{
Validation of the Portuguese version of an instrument \\ to measure the degree of patient knowledge about their medication
}

\author{
Joaquín Salmerón Rubio ${ }^{1}$ \\ Pilar García-Delgado ${ }^{1}$ \\ Paula Iglésias Ferreira ${ }^{2}$ \\ Henrique Mateus Santos ${ }^{2}$ \\ Fernando Martínez-Martínez ${ }^{1}$
}

\footnotetext{
${ }^{1}$ Grupo de Investigación en Atención Farmacéutica (CTS-131), Facultad de Farmacia, Universidad de Granada Granada, Campus de Cartuja. España FARJOA@teleline.es ${ }^{2}$ Grupo de Investigação em Cuidados Farmacêuticos (GICUF), Universidade Lusófona de Humanidades e Tecnologias (Lisboa).
}

\begin{abstract}
The scope of this study was the validation of a cross-culturally adapted questionnaire into Portuguese in five community pharmacies in Portugal. The discriminatory power of items, content and construct validity and factor analysis of the main components and their reliability and stability were determined. A high degree of semantic equivalence between the original questionnaire and the cross-culturally adapted questionnaire into Portuguese was observed. A Kaiser-Meyer-Olkin index of 0.550 was obtained and the Bartlett sphericity test confirmed the adequa$c y$ of the data for the application of factor analysis $(p<0.0001)$. Three factors which accounted for $52.6 \%$ of the total variability were considered. With respect to reliability the following results were obtained: 0.519 for Cronbach's alpha test; 0.89 for Cohen's kappa coefficient; and 0.756 (IC=0.598-0.963) for the CCI exam. In this work, the first adaptation for the Portuguese culture of a specific questionnaire was produced to measure the degree of knowledge patients have about their medication.
\end{abstract}

Key words Validation, Questionnaires, Patients' knowledge about their medication, Pharmaceutical practice, Pharmaceutical care
Resumen Validación de un cuestionario adaptado transculturalmente al portugués en cinco farmacias comunitarias de Portugal. Se determino la capacidad discriminante de los items, la validez de contenido y de constructo, análisis factorial de componentes principales, la fiabilidad y su estabilidad. Se observó un elevado grado de equivalencia semántica entre el cuestionario original y el cuestionario adaptado transculturalmente al portugués. Se obtuvo un indice de Kaiser-MeyerOlkin de 0.550, y el test de esferidad de Bartlett confirmó la adecuación de los datos para la aplicación del análisis factorial $(p<0.0001)$. Se consideraron 3 factores que explicaron el $52.6 \%$ de la variabilidad total. Con respecto a la fiabilidad se obtuvo un á de Cronbach de 0.519, un C.Kappa = 0.89 y CCI $=0.756(I C=0.598-0.963)$. En este trabajo se elabora la primera adaptación para la cultura portuguesa de un cuestionario específico para medir el grado de conocimiento que tienen los pacientes sobre los medicamentos.

Palabras clave Validación, Cuestionarios, Conocimiento del paciente sobre su medicamento, Práctica farmacéutica, Atención farmacéutica 


\section{Introducción}

La falta de conocimiento del paciente sobre su medicamento (CPM) parece que es un factor de riesgo para la aparición de resultados negativos asociados a la medicación (RNM), ya que puede conducir a un proceso de uso incorrecto, aumentando la probabilidad de que el medicamento no alcance su objetivo terapéutico o que origine nuevos problemas de salud. La morbimortalidad relacionada con el uso de los medicamentos tiene tal prevalencia que es un auténtico problema de salud pública ${ }^{1-5}$.

La falta de CPM merma la calidad del proceso general de atención al paciente, disminuyendo la probabilidad de obtener resultados clínicos positivos $^{6,7}$.

No existen demasiados datos publicados sobre el conocimiento de los pacientes en relación a sus medicamentos, y, los pocos estudios publicados miden diferentes aspectos del conocimiento y/o determinados grupos terapéuticos, sin contar, en la mayoría de ellos con una herramienta validada $^{8-19}$. La medida de un concepto tan abstracto y complejo, como el CPM, precisa de una herramienta que asegure que determina lo que se pretende medir, es decir, sea válida y que dé lugar a resultados reproducibles, es decir, sea fiable. Por lo que es necesario llevar a cabo un proceso riguroso de diseño y validación ${ }^{20}$.

Se entiende como CPM: "El conjunto de información adquirida por el paciente sobre su medicamento necesaria para un correcto uso del mismo, que incluye el objetivo terapéutico (indicación y efectividad), el proceso de uso (posología, pauta, forma de administración y duración del tratamiento), la seguridad (efectos adversos, precauciones, contraindicaciones e interacciones) y su conservación" 21 .

Se ha encontrado un único cuestionario genérico validado para medir el conocimiento de cualquier paciente con cualquier patología acerca de cualquier medicamento que utilice, el CPM de García-Delgado et al. ${ }^{21}$, validado en español.

Según un estudio realizado en dos provincias españolas este cuestionario válido y fiable (17), dos de cada tres pacientes no conocen el medicamento que utilizan, tan sólo el $9 \%$ presenta un conocimiento suficiente, y un $25 \%$ tienen un conocimiento óptimo de su medicación ${ }^{22}$.

Para poder utilizar en Portugal esta herramienta validada en español, es necesario cubrir un proceso metodológico riguroso que asegure que traducida al portugués sigue siendo válida y fiable. La primera fase es realizar una adaptación transcultural. Una vez obtenido el cuestionario traducido y adaptado culturalmente (CPM-PT$\mathrm{PT})^{23}$, se debe comprobar que la adaptación posee las mismas cualidades psicométricas que el instrumento original. En la adaptación transcultural de un instrumento, si el investigador demuestra de forma empírica que la versión traducida está estrechamente correlacionada con la versión original, es posible conferir a la versión traducida una validez concomitante, una validez de contenido a la vez, y una fiabilidad ${ }^{24-27}$.

Dado que el cuestionario validado ${ }^{21}$ contiene una escala de medida del CPM, con el que poder comparar valores de CPM en diferentes individuos o en el mismo individuo en diferentes momentos, se debe asegurar que el instrumento de medida adaptado transculturalmente al portugués arroje también un valor válido y fiable. Teniendo en cuenta que la validez del cuestionario es el grado en que un instrumento de medida sirve para el propósito para el que ha sido construi$\mathrm{do}^{28}$. A pesar de que se describen diferentes tipos de validez, ésta es un proceso unitario y permitirá realizar las inferencias e interpretaciones correctas de las puntuaciones que se obtengan y establecer la relación con el constructo/variable que se trata de medir ${ }^{29}$. Y la Fiabilidad es el grado en que un instrumento mide con precisión, sin error, se valora a través de la consistencia interna, la estabilidad temporal y la equivalencia ${ }^{8}$.

La finalidad del estudio es evaluar las propiedades psicométricas del cuestionario CPM adaptado transculturalmente al portugués europeo.

\section{Objetivo}

Validar la traducción intercultural del cuestionario "Conhecimento do doente sobre os seus medicamentos" (CPM-PT-PT) en portugués del cuestionario de Garcia-Delgado et al "Conocimiento del Paciente sobre sus Medicamentos" $(\mathrm{CPM}-\mathrm{ES}-\mathrm{ES})^{21}$.

\section{Método}

Para la estimación de la validez y fiabilidad del cuestionario, considerando un IC del 95\% y una precisión de $\pm 5 \%$, se establece un tamaño muestral óptimo de 100 sujetos. La selección de los participantes se realizó mediante muestreo consecutivo.

Se cumplimentó un cuestionario por paciente y medicamento en cinco farmacias comunita- 
rias de Portugal ubicadas en diferentes regiones de Portugal entre 29 de noviembre y el 29 de diciembre del 2009.

El cuestionario "Conhecimento do doente sobre os seus medicamentos" (CPM-PT-PT) 23 consta de 11 preguntas centrales, abiertas, que recogen el conocimiento del paciente acerca de su medicamento, determinando en cada pregunta un ítem básico del CPM: P.1. Indicación (Para que tem que tomar/utilizar este medicamento?); P.2. Posología (Quanto deve tomar/utilizar deste medicamento?); P.3. Pauta (Quando tem que tomar/utilizar este medicamento?); P.4. Duración del tratamiento (Até quando tem que tomar/utilizar este medicamento?); P.5. Forma de administración (Como debe tomar/utilizar este medicamento?); P.6. Precauciones (Deve ter alguma precaução quando toma/utiliza este medicamento?); P.7. Reacciones adversas (Que efeitos adversos conhece deste medicamento?); P.8. Contraindicaciones (Em que situações ou casos não deve tomar/utilizar este medicamento?); P.9. Indicadores de efectividad (Como é que sabe se o medicamento lhe faz efeito?); P.10. Interacciones (Que medicamentos ou alimentos deve evitar enquanto usa este medicamento?); P.11. Conservación (Que cuidados deve ter para manter em bom estado de conservação o seu medicamento?).

Además, el cuestionario contiene 12 preguntas adicionales, de las que 5 recogen las características sociodemográficas del paciente (edad, género, profesión, nivel de estudios, importancia para el paciente de su enfermedad) y las 7 restantes características relacionadas con el medicamento (grupo terapéutico, forma farmacéutica, no de medicamentos que toma, usuario del medicamento, conocimiento del nombre del medicamento, prescriptor, tiempo de uso del medicamento.

Cada cuestionario fue cumplimentado a través de entrevista personal farmacéutico-paciente, seleccionando un medicamento por paciente. La evaluación de las preguntas de conocimiento sobre el medicamento (de P.1. a P.11.), se llevó a cabo por tres farmacéuticos entrenados para tal fin, que contrastaron las respuestas dadas por el paciente con la información de referencia (la receta médica y/o los RCM de Infarmed o de la EMA (European Medicines Agency) y asignaron a cada respuesta, para cada pregunta, la siguiente puntuación en función del grado de concordancia entre la información dada por el paciente y la información de referencia:

. Información incorrecta: -1 (La información del paciente no coincide con la información de referencia);
. No conoce: 0 (El paciente comunica verbal o no verbalmente que no sabe);

. Información insuficiente: 1 (La respuesta del paciente es incompleta o que no posea la información necesaria para asegurar un correcto proceso de uso);

. Información correcta: 2 (La información del paciente no coincide con la información de referencia).

Para el cómputo final del conocimiento total del medicamento (CPM global) se aplicó una fórmula en la que cada pregunta puntuaba diferente en base a las conclusiones extraídas en las distintas técnicas cualitativas. El CPM global se categorizó en:

- No conoce. 0 puntos;

- Conocimiento insuficiente. De 0.60 a 1.26 puntos;

- Conocimiento suficiente. De 1.27 a 1.60 puntos;

- Conocimiento óptimo. De 1.60 a 2 puntos.

El análisis estadístico de los datos se realizó de acuerdo a las siguientes pruebas: capacidad discriminante de los ítems, validez de constructo y fiabilidad.

A. Capacidad discriminante de los ítems: Se estimó mediante la frecuencia de endose y el coeficiente de correlación de Pearson.

B. Validez:

a) La validez de contenido se estimó en el proceso de adaptación transcultural mediante la comisión de expertos y la revisión bibliográfica.

b) Las pruebas estadísticas utilizadas para analizar la validez de constructo fueron: Análisis de correlaciones entre las distintas medidas de conocimiento del estudio mediante el cálculo del coeficiente Rho de Spearman y el Análisis Factorial de Componentes Principales (AFCP) para estudiar la dimensionalidad del concepto "Conocimiento sobre el medicamento".

Para calcular el AFCP se llevaron a cabo las siguientes etapas:

1. Cálculo y examen de la matriz de correlaciones:

. Test de esfericidad de Barlett.

. Índice de Kaiser-Meyer-Olkin (KMO).

2. Aplicación del método de componentes principales: por medio del cual se exploraron las posibilidades de reducir la información mediante la construcción de un nuevo conjunto de factores a partir de las interrelaciones observadas en los datos.

3. Rotación de los factores: Se utilizó el método de rotación Normalización Varimax con Kaiser. 
4. Interpretación de los factores: durante esta etapa se estudió la composición de las cargas factoriales significativas de cada factor, y se les nombró. Se empleó el método Kaiser-Guttman de extracción de factores con eigenvalues (valores propios o autovalores) superiores a 1 .

C. Fiabilidad:

a) La consistencia interna $u$ homogeneidad del instrumento se valoró calculando el coeficiente Alpha de Cronbach.

b) Equivalencia: Se aplicó el índice de concordancia inter-observadores, índice Kappa. Este valor se obtuvo al ser evaluado por 3 investigadores diferentes, todos los cuestionarios del estudio piloto, y comparar los resultados obtenidos por cada evaluador para cada uno de los cuestionarios.

c) Con el fin de determinar la estabilidad del cuestionario se realizó la prueba test-retest que se determinó mediante el coeficiente de correlación intraclase (CCI) ${ }^{30-32}$. Para ello se seleccionaron durante 2 meses, en cinco farmacias de diferentes regiones de Portugal, los pacientes que tuvieran un conocimiento suficiente del medicamento, el que se volvió a medir transcurridos 20 30 días.

\section{Resultados}

Se incluyeron en el estudio un total de 100 pacientes, el 65\% fueron mujeres, con edades comprendidas entre 20 y 87 años. El tiempo medio para completar la entrevista fue de 6,8 minutos $(\mathrm{DE}=3,9)$, con un rango de 3 a 29 minutos. Las características de los pacientes del estudio se muestran en la Tabla 1.

Capacidad discriminante de los ítems del cuestionario adaptado transculturalmente al portugués.

La distribución de frecuencias absolutas para cada una de las 11 preguntas relacionadas con el CPM adaptado transculturalmente al portugués europeo varía del 0 al $82 \%$ tal como muestra la Tabla 2.

Cabe destacar tres preguntas en las que el valor de saturación ha sido superior al 75\%; estas preguntas son la P.1 (Indicación) con un $82 \%$ en la opción “Conoce”, la P.2 (Posología) con un 79\% y la P.9 (Efectividad) con un $76 \%$ para la misma categoría. Es destacable el porcentaje obtenido para la opción "Conoce" de la P.8 (Contraindicaciones) que solo obtiene un porcentaje del $1 \%$.

Se observa que solo una categoría no está representada, obtiene un $0 \%$ las respuestas catego- rizadas como "información insuficiente" para la P. 3 (Pauta), siendo del 1\% para la categoría "información insuficiente” de la pregunta P.1 (Indicación) y P.2 (Posología). Para la categoría "No conoce” la pregunta P.12 (Conservación) presen-

Tabla 1. Resumen global de las características de los pacientes incluidos en el estudio piloto ( $\mathrm{n}^{\circ}$ de pacientes $=100)$.

\begin{tabular}{|c|c|c|}
\hline & $\mathbf{n}$ & $(\%)$ \\
\hline \multicolumn{3}{|l|}{ Edad } \\
\hline 20-34 años & 27 & $(27,0)$ \\
\hline 35-49 años & 29 & $(29,0)$ \\
\hline 50-64 años & 19 & $(19,0)$ \\
\hline 65 o más años & 25 & $(25,0)$ \\
\hline \multicolumn{3}{|l|}{ Género } \\
\hline Hombre & 35 & $(35,0)$ \\
\hline Mujer & 65 & $(65,0)$ \\
\hline \multicolumn{3}{|l|}{ Profesión } \\
\hline Amas de casa/jubilados/paro & 33 & $(33,0)$ \\
\hline Trabajos manuales no cualificados & 7 & $(7,0)$ \\
\hline Trabajos manuales cualificados & 22 & $(22,0)$ \\
\hline Administrativos y comerciantes & 22 & $(22,0)$ \\
\hline Universitarios & 8 & $(8,0)$ \\
\hline Clase dirigente y empresarios & 8 & $(8,0)$ \\
\hline \multicolumn{3}{|l|}{ Nivel de estudios } \\
\hline No sabe leer ni escribir & 2 & $(2,0)$ \\
\hline Sabe leer y escribir (sin estudios) & 1 & $(1,0)$ \\
\hline 1er ciclo de ensino básico & 32 & $(32,0)$ \\
\hline 2do ciclo de ensino básico & 3 & $(3,0)$ \\
\hline 3er ciclo de ensino básico & 14 & $(14,0)$ \\
\hline Ensino secundario & 28 & $(28,0)$ \\
\hline Ensino superior & 20 & $(20,0)$ \\
\hline \multicolumn{3}{|l|}{ Número de medicamentos que toma } \\
\hline Monoterapia & 24 & $(24,0)$ \\
\hline De 2 a 4 medicamentos & 47 & $(47,0)$ \\
\hline Polimedicado (5 o más) & 29 & $(29,0)$ \\
\hline \multicolumn{3}{|l|}{ Usuario del medicamento } \\
\hline Uso propio & 71 & $(71,0)$ \\
\hline Cuidador & 29 & $(29,0)$ \\
\hline \multicolumn{3}{|l|}{ Prescriptor del medicamento } \\
\hline Médico & 96 & $(96,0)$ \\
\hline Farmacéutico & 0 & $(0)$ \\
\hline Otros & 4 & $(4,0)$ \\
\hline \multicolumn{3}{|l|}{ Tiempo de uso del medicamento } \\
\hline Primera prescripción & 30 & $(30,3)$ \\
\hline De 0,5 a 6 meses & 23 & $(23,2)$ \\
\hline De 7 a 12 meses & 6 & $(6,1)$ \\
\hline De 13 a 24 meses & 6 & $(6,1)$ \\
\hline Más de 24 meses & 34 & $(34,3)$ \\
\hline \multicolumn{3}{|l|}{$\begin{array}{l}\text { Importancia para el paciente de su } \\
\text { enfermedad }\end{array}$} \\
\hline Poco & 29 & $(29,0)$ \\
\hline Regular & 38 & $(38,0)$ \\
\hline Bastante & 33 & $(33,0)$ \\
\hline
\end{tabular}


Las correlaciones de Spearman entre ítems fueron en general bajas, si bien en algunos casos se encontraron correlaciones superiores a 0,30 que resultaron significativas. Las variables que mejor correlación mostraron fueron pauta con posología (0.709) y la forma de administración con posología y pauta ( 0,362 y 0,312 respectivamente).

Con respecto al Análisis Factorial de Componentes Principales (AFCP) se obtuvo un índice de Kaiser-Meyer-Olkin de 0,550 y el test de esferidad de Bartlett confirmó la adecuación de los datos para la aplicación del análisis factorial $(\mathrm{p}<$ 0.0001 ). La medida de adecuación muestral para cada ítem varió entre 0,451 y 0,653 (Tabla 3 ).

La mayoría de los valores fueron superiores a 0,40 excepto para las preguntas P.1 (Indicación), P.7 (Efectos Adversos) y P.11 (Conservación). En el caso del análisis de componentes principales no se supone la existencia de ningún factor común, por lo que la nominalidad inicial toma el valor 1 .

El análisis de dimensionalidad del constructo "CPM" ha mostrado una estructura factorial clara. Se observa que el AFCP reveló 4 componentes (usando el criterio de un eigenvalue mayor que 1) explicando el $54,084 \%$ de la varianza total. Se realizó un análisis factorial con el método de extracción de componentes principales excluyendo aquellos ítems con una medida de adecuación muestral inferior a 0,5 que se considera inacepta-

ble para la aplicación de estas técnicas multivariantes. En base a este criterio no se incluyeron en el análisis factorial los ítems "P6-Precauciones" y "P10-Interacciones".

Se consideraron 3 factores con autovalores mayores de 1, que explicaron el 52,6\% de la variabilidad total (Tabla 4). La estructura factorial rotada mediante el método Varimax mostró 3 dimensiones claramente diferenciadas:

Primera dimensión, compuesta por los ítems: "P2-Posología", "P3-Pauta" y "P5-Forma de administración".

Segunda dimensión, compuesta por los ítems "P1-Indicación", "P4-Duración del tratamiento", "P9-Efectividad" y "P11-Conservación".

Tabla 3. Medida de adecuación muestral de cada ítem e índice de Kaiser-Meyer-Olkin.

\begin{tabular}{lc}
\hline & $\begin{array}{c}\text { Medida de } \\
\text { adecuación } \\
\text { muestral }\end{array}$ \\
\hline P1 Indicación & 0,554 \\
P2 Posología & 0,530 \\
P3 Pauta & 0,552 \\
P4 Duración del tratamiento & 0,546 \\
P5 Forma de administración & 0,653 \\
P6 Precauciones & 0,463 \\
P7 Efectos adversos & 0,577 \\
P8 Contraindicaciones & 0,517 \\
P9 Efectividad & 0,612 \\
P10 Interacciones & 0,451 \\
P11 Conservación & 0,549 \\
KMO global & 0,550 \\
\hline
\end{tabular}

Tabla 2. Frecuencia de endose para cada ítem del cuestionario de conocimiento del paciente sobre su medicamento.

\begin{tabular}{lcccc}
\hline & Conoce & Información insuficiente & Información incorrecta No conoce \\
\hline P.1. Indicación & 82 & 1 & 10 & 7 \\
P.2. Posología & 79 & 1 & 6 & 14 \\
P.3. Pauta & 75 & 0 & 14 & 11 \\
P.4. Duración del tratamiento & 64 & 2 & 11 & 23 \\
P.5. Forma de administración & 70 & 3 & 15 & 12 \\
P.6. Precauciones & 9 & 16 & 60 & 15 \\
P.7. Efectos adversos & 8 & 14 & 13 & 65 \\
P.8. Contraindicaciones & 1 & 12 & 19 & 68 \\
P.9. Efectividad & 76 & 5 & 6 & 13 \\
P.10. Interacciones & 5 & 18 & 20 & 57 \\
P.11. Conservación & 10 & 82 & 6 & 2 \\
\hline
\end{tabular}


Tercera dimensión, compuesta por los ítems "P7 Efectos adversos" y "P8 Contraindicaciones".

\section{Fiabilidad del cuestionario}

El coeficiente Alpha de Cronbach para el conjunto de ítems fue 0,519. Los resultados obtenidos en cuanto a la equivalencia del cuestionario, que se midió por el coeficiente Kappa ponderado, muestran un elevado grado de concordancia inter-observadores $(0,89)$ que varió entre 0,473 (IC95\% 0,57-0,76) para el ítem "P6-Precauciones” y 0,944 (IC95\% 0,85-1,00) para “P7-Efectos adversos". La concordancia del conocimiento global fue de 1 (Tabla 5). El coeficiente más bajo se

Tabla 4. Matriz de componentes rotados del análisis factorial.

\begin{tabular}{lccc}
\hline & \multicolumn{3}{c}{ Factores } \\
\cline { 2 - 4 } & I & II & III \\
\hline P1 Indicación & \multicolumn{3}{c}{0,580} \\
P2 Posología & 0,875 & & \\
P3 Pauta & 0,877 & & \\
P4 Duración del tratamiento & & 0,453 & \\
P5 Forma de administración & 0,535 & & \\
P7 Efectos adversos & & & 0,553 \\
P8 Contraindicaciones & & & 0,669 \\
P9 Efectividad & & 0,721 & \\
P11 Conservación & & 0,539 & \\
Autovalor & 2,068 & 1,457 & 1,202 \\
\% de variabilidad explicada & $23,0 \%$ & $16,2 \%$ & $13,4 \%$ \\
\hline & & & \\
\hline
\end{tabular}

obtuvo para el ítem "P9-Efectividad" $(0,469)$, y se alcanzó un grado de concordancia del 100\% para 5 de los 11 ítems del cuestionario. El coeficiente de correlación intraclase para la puntación del conocimiento global del medicamento fue de 0,756 (IC95\% 0,548-0,963).

\section{Discusión}

Es importante señalar que la muestra de pacientes con la que se realizó la validación del cuestionario se obtuvo mediante un muestreo consecutivo en vez de efectuar una técnica probabilística, ya que el objetivo fundamental era efectuar la valoración de las propiedades psicométricas del cuestionario. Por ello no pueden generalizarse los datos obtenidos, ni siquiera a la totalidad de los pacientes que concurrieron a la farmacia de estudio. En este sentido, es imposible sostener que los resultados referentes al CPM tienen validez externa. Es decir, los resultados obtenidos no se pueden extrapolar a la población general de la que fue extraída la muestra. Sin embargo, se persiguió que los pacientes seleccionados fuesen lo más heterogéneos posibles; diferentes edades, nivel social-económico, nivel de estudios, patologías, medicamentos utilizados; con el fin de evitar el sesgo de selección ${ }^{33}$.

Con respecto al AFCP, mostró que el conocimiento del paciente sobre el medicamento que utiliza no es un concepto unidimensional, sino multidimensional. Estos datos se han podido comparar con los obtenidos por García-Delgado et $a .^{21}$, en español, siendo la estructura del

Tabla 5. Análisis de concordancia inter-observadores. Coeficiente Kappa de cada ítem y el conocimiento global $\left(n^{\circ}\right.$ de pacientes $=100 ; n^{\circ}$ de observadores $=3$ ).

\begin{tabular}{lcccc}
\hline \multicolumn{1}{c}{ Ítems } & Coeficiente Kappa & Valor $\mathbf{p}$ & I.C. $(\mathbf{9 5} \%)$ & $\mathbf{N}$ \\
\hline P.1. Indicación & 0,911 & $<0,001$ & $(0,780-1,000)$ & 100 \\
P.2. Posología & 0,86 & $<0,001$ & $(0,640-1-000)$ & 100 \\
P.3. Pauta & 0,777 & $<0,001$ & $(0,501-1,000)$ & 100 \\
P.4. Duración del tratamiento & 0,92 & $<0,001$ & $(0,850-0,997)$ & 100 \\
P.5. Forma de administración & 0,626 & $<0,001$ & $(0,368-0,773)$ & 100 \\
P.6. Precauciones & 0,473 & $<0,001$ & $(0,571-0,763)$ & 100 \\
P.7. Efectos adversos & 0,944 & $<0,001$ & $(0,848-1,000)$ & 100 \\
P.8. Contraindicaciones & 0,670 & $<0,001$ & $(0,772-0,928)$ & 100 \\
P.9. Efectividad & 0,924 & $<0,001$ & $(0,862-0,992)$ & 100 \\
P.10. Interacciones & 0,728 & $<0,001$ & $(0,798-0,952)$ & 100 \\
P.11. Conservación & 0,676 & $<0,001$ & $(0,717-0,908)$ & 100 \\
CMT (4 categorías) & 1 & - & - & 100 \\
CMT (2 categorías) & 1 & - & - & 100 \\
\hline
\end{tabular}


conocimiento hallada en el cuestionario desarrollado coincidente con el argumento del marco teórico. El análisis de dimensionalidad del constructo "conocimiento sobre el medicamento por el paciente" ha mostrado una estructura factorial clara.

El coeficiente Alpha de Cronbach de la escala fue de 0,5 , que según algunos autores se puede considerar un valor aceptable. El motivo por el que se obtuvo un valor poco elevado puede estar relacionado con el pequeño tamaño de la muestra, no obstante se puede confirmar que el cuestionario diseñado es fiable con respecto a la homogeneidad de la escala. La homogeneidad total del cuestionario adaptado al portugués (europeo) no mejoraría al eliminar alguno de los ítems.

Una de las limitaciones del estudio es la evaluación de los datos. La asignación de las diferentes puntuaciones parciales de cada ítem se ha realizado teniendo como referencia las puntuaciones de García-Delgado et al. ${ }^{21}$ utilizadas en la evaluación del cuestionario original en español, con el fin de poder hacer un estudio comparativo en los diferentes países, lo que supone una carga de subjetividad importante que pudo afectar la validez interna del estudio al utilizar esta herramienta como instrumento de medida. Para reducir este efecto se propuso unas normas de evaluación, que implicó una formación previa a los evaluadores con el fin de unificar criterios de valoración. La forma de evaluar también afecta a la equivalencia de este y por lo tanto a su fiabilidad.

Con el fin de determinar si el cuestionario adaptado transculturalmente al portugués era equivalente se midió el coeficiente Kappa ponderado, mostrando un elevado grado de concordancia inter-observadores. También se determinó el coeficiente Kappa ponderado para cada uno de los ítems evaluados por ambos observadores y el coeficiente Kappa ponderado para el CPMPT-PT para cada paciente evaluado por los observadores. La concordancia inter-observadores varió entre 0,473 (IC95\% 0,57-0,76) para el ítem “P6-Precauciones" y 0,944 (IC95\% 0,85-1,00) para "P7-Efectos adversos".

La concordancia del conocimiento global fue de 1 , lo que indica que el conocimiento del paciente sobre su medicamento es una medida susceptible de variar en el tiempo. El poder hallar diferencias en el conocimiento del paciente sobre su medicación después de una intervención sanitaria nos muestra la capacidad del instrumento para detectar la sensibilidad al cambio. Para determinar la estabilidad del cuestionario adapta- do transculturalmente al portugués se hizo mediante test-retest.

Debido a que los farmacéuticos entrevistadores no podían corregir, aumentar o modificar el conocimiento medido del paciente sobre su medicamento, se optó por seleccionar para esta prueba solo aquellos pacientes que presentasen un conocimiento suficiente, como mínimo, evitando posibles problemas éticos motivados por la ausencia de intervención educativa. El coeficiente más bajo se obtuvo para el ítem "P9-Efectividad" $(0,469)$, y se alcanzó un grado de concordancia del $100 \%$ para 5 de los 11 ítems del cuestionario. El coeficiente de correlación intraclase para la puntación del conocimiento global del medicamento fue de 0,756 (IC95\% 0,548$0,963)$. Es posible que el paciente al sentirse interrogado, considerase la necesidad de recurrir a la lectura del prospecto u otra información de referencia, pudiendo modificar su conocimiento.

En base a los resultados obtenidos referentes, a la consistencia interna, equivalencia y fiabilidad del cuestionario adaptado transculturalmente al portugués, es una herramienta útil, ágil, válida y fiable para cuantificar el nivel básico de conocimiento de los pacientes sobre sus medicamentos.

Aunque en aquellos estudios en la que aspectos sociales ${ }^{34,35}$, es decir donde haya una relación entre el conocimiento y comportamiento tengan que medirse de manera detallada y por separado, este cuestionario adaptado transculturalmente al portugués europeo debería completarse con otras preguntas o escalas especificas para cada una de las dimensiones, ya que un solo ítem es insuficiente para abarcar una sola dimensión como es la "conservación", así como otras dimensiones deberían abarcar mayor número de ítems para evitar el sesgo importante entre conocimiento y comportamiento que tienen los pacientes sobre sus medicamentos. La utilización del cuestionario tanto para la práctica clínica como para la evaluación de las diferentes intervenciones sanitarias portuguesas, hace necesario el seguimiento y consenso de las normas de evaluación para evitar también sesgos de clasificación.

\section{Conclusiones}

La similitud de las características operacionales como la consistencia interna, la reproducibilidad y la validez de la versión portuguesa apoya la hipótesis de que las versiones portuguesas y españolas del CMP son conceptualmente equivalentes. No obstante, se hace necesario desarrollar 
nuevos estudios con mayor tamaño de la muestra, establecer normas de evaluación específicas, además de evaluar otras características psicométricas como la sensibilidad a los cambios en el conocimiento sobre los medicamentos que tengan los pacientes después de recibir una intervención sanitaria en la que exista información del medicamento al paciente. En base a los resultados obtenidos referentes, a la consistencia interna, equivalencia y fiabilidad del cuestionario adaptado transculturalmente al portugués, es una herramienta útil, ágil, válida y fiable para cuantificar el nivel básico de conocimiento de los pacientes sobre sus medicamentos.

\section{Colaboradores}

JS Rubio ha trabajado en la concepción, investigación y en la metodología; PI Ferreira y PG Delgado en la concepción, metodología de investigación y estadística; HM Santos en la investigación, la redacción y la metodología, FM Martínez en la concepción y en la metodología.

\section{Agradecimientos}

Beca de Investigación para realizar la Tesis Doctoral a desarrollar en Universidad Lusofona, Lisboa, Portugal, por convenio entre Colegio Oficial de Farmacéuticos de la Región de Murcia y Cajamurcia, bajo la dirección de la Universidad de Granada. 


\section{Referencias}

1. Johnson JA, Bootman JL. Drug-related morbidity and mortality. A cost-of-illness Model. Arch Intern Med 1995; 155(18):1949-1956.

2. Ernst FR, Grizzle AJ. Drug-related morbidity and mortality: updating the cost-of-illness model. $J \mathrm{Am}$ Pharm Assoc (Wash) 2001; 41(2):192-199.

3. Climente M, Quintana I, Martinez G, Atienza A, Jiménez NV. Prevalencia y características de la morbilidad relacionada con los medicamentos como causa de ingreso hospitalario. Aten Farm 2001; 3(1):9-22.

4. Baena MI, Faus MJ, Fajardo PC, Luque FM, Sierra F, Martinez-Olmos J, Cabrera A, Fernandez-Llimos F, Martinez-Martinez F, Jiménez J, Zarzuelo A. Medicine-related problems resulting in emergency department visits. Eur J Clin Pharmacol 2006; 62(5):387-393.

5. Correa AD, Caminha J, Alves L. Uma abordagem sobre o uso de medicamentos nos livros didáticos de biologia como estratégia de promoção de saúde. Cien Saude Colet 2013; 18(10):3071-3081.

6. DeVries TPGM, Henning RH, Hogerzeil HV, Fresle DA. Guide to good prescribing. Geneva: WHO/Action programme on essential drugs; 1994.

7. DeVries TPGM. Presenting clinical pharmacology and therapeutics: A problem based approach for choosing and prescribing drugs. Br J Clin Pharmacol 1993; 35(6):581-586.

8. American College of Emergency Physicians. Study Finds Elderly Patients Know Too Little About Their Medications. [Internet]. [cited 2009 Nov 11]. Available from: http://www.eurekalert.org/pub_releases/ 2002-05/acoe-sfe053002.php

9. Boonstra E, Lindbaek M, Ngome E, Tshukudu K, Fugelli P. Labelling and patient knowledge of dispensed drugs as quality indicators in primary care in Botswana. Qual Saf Health Care 2003; 12(3):168-175.

10. Hughes L, Wittlesea C, Luscombe D. Patients' knowledge and perceptions of the side-effects of OTC medication. J Clin Pharm Ther 2002; 27(4):243-248.

11. O'Connell MB, Johnson JF. Evaluation of medication knowledge in elderly patients. Ann Pharmacother 1992; 26(7-8):919-921.

12. Huang YM, Wang HP, Yang YH, Lin SJ, Lin HW, Chen CS, Wu FL. Effects of a national health education program on the medication knowledge of the public in Taiwan. Ann Pharmacother 2006; 40(1):102108.

13. Silva T SE, Mengue SS. Nivel de informação respeito de medicamentos prescritos a pacientes ambulatoriais de hospital universitário. Cad Saude Publica 2000; 16(2):449-455.

14. Menolli PVS, Ivama AM, Cordoni Júnior L. Caracterización de los servicios farmacéuticos de atención primaria del Sistema Único de Salud en Londrina, Paraná, Brasil. Rev Panam Salud Publica 2009; 25(3):254-259.

15. Silva Td, Dal-Pizzol F, Bello CM, Sotero Serrante M, Schenkel EP. Bulas de medicamentos e a informação adequada ao paciente. Rev Saude Publica 2000; 34(2):184-189.
16. Frohlich SE, Dal-Pizzol Tda S, Mengue SS. Instrumento para avaliação do nível de conhecimento da prescrição na atenção primária. Rev Saude Publica 2010; 44(6):1046-1054.

17. Holanda WTG, de Lima MLC, Figueiro JN. Adaptação transcultural de um instrumento de avaliação do handicap auditivo para portadores de perda auditiva induzida pelo ruído ocupacional. Cien Saude Colet 2011; 16(Supl. 1):755-767.

18. da Franca C, Colares V. Validação do National College Health Risk Behavior Survey para utilização com universitários brasileiros. Cien Saude Colet 2010; 15(Supl. 1):1209-1215.

19. Cruzeta A, Oro AC, Mattos MT, Martins RO, Calegario TA, Galato D. Fatores associados à compreensão da prescrição médica no Sistema Único de Saúde de um município do Sul do Brasil. Cien Saude Colet 2013; 18(12):3731-3737.

20. Laurencelle L. Théorie et techniques de la mesure instrumentale. Montreal: Presses de l'Université du Québec; 1998.

21. García-Delgado P, Gastelurrutia MA, Baena MI, Fisac F, Martínez-Martínez F. Validación de un cuestionario para medir el conocimiento de los pacientes sobre sus medicamentos. Aten Primaria 2009; 41(12):661-669.

22. García-Delgado P, Martínez-Martínez F. Instrumento de medida, prevalencia y factores asociados al conocimiento. $1^{\text {a }}$ ed. Saarbrücken: Editorial Académica Española; 2012.

23. Salmerón-Rubio J, Iglésias-Ferreira P, Delgado PG, Mateus-Santos H, Martínez-Martínez F. Adaptação intercultural para português europeu do questionário "Conocimiento del Paciente sobre sus Medicamentos" (CPM-ES-ES). Cien Saúde Colet 2013; 18(12):3633-3644

24. Vallerand RJ, Halliwell WR. Vers une methodologie de validation trans-culturelle de questionnaires psychologiques: Implications pour la psychologie du sport. Can J Applied Sport Sci 1983; 8(1):9-18.

25. Nunnaly JC, Bernstein IJ. Validez. New York: Ed. McGraw Hill; 1995. p. 92-127.

26. Iglésias-Ferreira P, Santos HJ, Fernández-Llimós F, Fontes E, Leal M, Monteiro C. Traducción y Validación del "Pharmacy Services Questionnaire" al portugués (europeo). Seguim Farmacoter 2005; 1(3): 43-56.

27. Santos H, Iglésias-Ferreira P, Fernández-Llimós F, Faus MJ, Rodrigues LM. Segundo Consenso de Granada sobre Problemas Relacionados com Medicamentos - Tradução Intercultural de Espanhol para Português (europeu). Acta Médica Portuguesa 2004; 17(1):59-66.

28. Azofra MJ. Cuestionarios. Cuadernos metodológicos. Madrid: Centro de Investigaciones Sociológicas; 1999.

29. Goode WJ, Hato PK. Métodos de investigacion social. Mexico: Trillas; 1981. 
30. Prieto L, Lamarca R, Casado A. La evaluación de la fiabilidad en las observaciones clínicas: el coeficiente de correlación intraclase. Med Clin (Barc) 1998; 110(4):142-145.

31. Pita Fernandéz S, Pértegas Díaz S. La fiabilidad de las mediciones clínicas: el análisis de concordancia para variables numericas. [Internet]. [cited 2009 Nov 16]. Available from: http://www.fisterra.com/ mbe/investiga/conc_numerica/conc_numerica.asp.

32. Rodriguez C, Sossa MP. Validación de un cuestionario de conocimientos acerca del asma entre padres o tutores de niños asmáticos. Arch Bronconeumol 2005; 41(8):419-424.

33. Streiner DL, Norman GR. Consistencia, validez y fiabilidad en las escalas de medida de la salud (Adaptado por Grau-Fibla G). In: Streiner DL, Norman GR, editors. Health measurement scales: a practical guide to their development and use. $2^{\text {nd }}$ ed. New York: Oxford University Press; 1992.

34. García Cadena C. La medición en ciencias sociales y en la psicología. In: Landeros Hernández, René y González Ramírez, Mónica T, editores. Estadística con SPSS y metodología de la investigación. México: Trillas; 2006.

35. Hogan Thomas P. Pruebas psicológicas. México: El Manual Moderno; 2004.

Artigo apresentado em 24/09/2012

Aprovado em 23/10/2012

Versão final apresentada em 05/11/2012 